\section{Acute Poisoning: Diagnosis and Management}

by A.T. Proudfoot. Butterworth Heinemann, London, 1993. £18.95, ISBN 0-7506-1445-5.

Accident and emergency departments see many adults and children with poisoning or suspected poisoning. Information about the possible problems and necessary treatment is needed rapidly. This book by Alex Proudfoot of Edinburgh Royal Infirmary provides such information. The first edition was published in 1982 and has been out of print for some years. This second edition is most welcome. The format is unchanged but the contents have been radically revised and up-dated. The initial chapters cover the classification and clinical diagnosis of poisoning and the laboratory investigations that may be needed. The general management of acute poisoning is discussed in detail. The advice about when (and when not) to use gastric lavage or ipecac will be helpful in avoiding unnecessary treatment. The main section of the book covers specific poisons, including all the common drugs, and also problems such as adder bites, button batteries and some poisonous plants and mushrooms. There are many references for further reading and a useful appendix on drug addicts' names for their favourite poisons.

The author's advice is sensible and practical, based on many years of experience in the Regional Poisoning Treatment Centre and the Scottish Poisons Information Bureau. This book is strongly recommended and is an essential reference book for accident and emergency departments.

R.N. ILLINGWORTH

Consultant in Accident and Emergency Medicine St James's University Hospital, Leeds

\section{External Eye Disease: A Colour Atlas}

by M.T. Watts \& M.E. Nelson. Churchill Livingstone, Edinburgh, 1992. 102 pp. £49.50. ISBN 0-44304446-5.

This 100-page book is a very precise descriptive atlas of the eyelids, the conjunctiva and the cornea. This is a detailed work primarily for the trainee ophthalmologist. However, it does, of course, cover the common conditions which are seen in any accident and emergency (A\&E) department, especially in the inner city. It presents clearly the pathology even if the text is for the specialist eye surgeon rather than for the generalist in A\&E medicine. It does make one wonder about a number of conditions that perhaps are not diagnosed correctly initially in the A\&E department. For instance, how often does a patient with adult inclusion conjunctivitis caused by chlamydia present to an A\&E department, remembering that most departments do not have the correct swabs for chlamydia?

It is, however, a pity that points relevant to A\&E medicine are not emphasized, e.g. the importance of staining the red eye for a possible dendritic ulcer and, further, the safe advice that A\&E departments should not prescribe steroid eye drops.

If this book had started from the differential diagnosis of the red eye it would be more user friendly for the non eye specialist. As it is, it is a reference book which would be of use to those doctors who work in A\&E departments who have a specialist interest in eyes. It does have a good index and could, perhaps, be of use when combined with a general reference text book of A\&E medicine, as it is, it is probably too specialized for most A\&E department libraries.

\section{R. TOUQUET}

Accident and Emergency Department, St. Mary's Hospital, Praed Street, London 